\title{
Rotavirus Infection Among Hospitalized Children With Gastroenteritis in A Teaching Hospital in Central Iran: A Cross-Sectional Study
}

Mohamad Amin Khajeh-Azad

Iran University of Medical Sciences

\section{Sajjad Rezvan}

Qom university of medical sciences

\section{Saeed Shams}

Qom university of medical sciences

\section{Zahra Movahedi}

Qom university of medical sciences

\section{Mohammad Aghaali}

Qom university of medical sciences

Hosein Heydari ( $\nabla$ hoseinheydari3375@yahoo.com )

Qom university of medical sciences

\section{Research Article}

Keywords: Rotavirus, acute gastroenteritis, diarrhea, children, Para clinic

Posted Date: July 20th, 2021

DOl: https://doi.org/10.21203/rs.3.rs-654085/v1

License: (9) (i) This work is licensed under a Creative Commons Attribution 4.0 International License. Read Full License 


\section{Abstract \\ Background}

Human Rotaviruses are important causative agent of acute gastroenteritis among young children globally. Therefore, the study of diarrheal samples obtained from children in order to determine the prevalence and dominant genotypes of Rotavirus is indispensable to prevent inappropriate prescription of antibiotics and to assess the necessity of vaccine immunization. We aimed to investigate the prevalence of Rotavirus infections among children with acute diarrhea and to study the association of the disease with paraclinical parameters in Qom, central Iran.

\section{Methods}

We collected stool samples from children admitted with acute diarrhea to a teaching hospital. EnzymeLinked-Immunosorbent Assay (ELISA) was used to detect Rotavirus antigens. Blood samples were taken to measure $\mathrm{CBC}$, ESR, and CRP levels.

\section{Findings:}

Rotavirus was detected in $21.4 \%$ (40/199) of the samples. The mean age of children in this study was $8 / 2 \pm 2 / 3$ years. There was a statistically significant difference between no increase in WBC, PMN, CRP and ESR in children with rotavirus infection.

\section{Conclusion}

Our study showed a lower prevalence of Rotavirus infections in hospitalized children compared to previous studies. We suggest that in addition to Rotavirus detection using PCR and genotyping, other viruses should be sought for detection of causative agent of diarrhea in children.

\section{Introduction}

Rotavirus, a double-stranded RNA virus from the Reoviridae family, is one of the major causes of gastroenteritis among children and teenagers globally. It can also cause encephalitis, exanthema subitum, otitis media, necrotizing enterocolitis and hepatic abscesses(1-4).

Symptomatic infection is most common in children aged 6 to 24 months, however, asymptomatic infection can occur at younger ages too (when the maternal antibodies are still in circulation)(5). Antirotavirus antibodies are highly prevalent among adults, probably due to asymptomatic re-infection. The virus is transmitted through the oral-fecal route, as well as iatrogenically(6). 
Studies show that 500 to 600 thousand children die annually due to Rotavirus infection, 85 percent of which are from low-income countries $(1,7,8)$. An effective vaccination program can reduce hospitalization rate and expenses $(8-10)$. Due to its high mortality and case-fatality rates $(8,11)$, the World Health Organization(WHO) has recommended hospital monitoring to identify common rotavirus genotypes in order to develop vaccines. Typing is based on two capsid proteins VP4 (P) and VP7 (G) (12). Rotavirus detection in stool samples is not a routine laboratory work-up and can be achieved using a variety of methods with different levels of diagnostic accuracy including enzyme-linked immunosorbent assay (ELISA), latex agglutination (LA), electron microscopy (EM), and molecular methods(13). Due to the lack of information about the prevalence of Rotavirus infection in Qom province, physicians usually treat diarrhea empirically. This approach leads to antibiotic over-prescription which in turn can increase antibiotic resistance where conservative treatment would suffice. This study aimed to investigate the prevalence of Rotavirus infection and its association with clinical and laboratory markers among hospitalized children with diarrhea.

\section{Materials And Methods}

\section{Patients and design}

We conducted a cross-sectional study of rotavirus prevalence among 199 patients hospitalized with diarrhea at Hazrat-e-Masoumeh pediatric hospital during a period of one year. We included children more than 1 month of age who were hospitalized with acute diarrhea. Individuals with bloody diarrhea were excluded from the study. All patients underwent laboratory testing including CBC, ESR, and CRP as well as stool examination for rotavirus infection using antigen( $\mathrm{Ag})$ detection method. The study protocol was approved at ethics committee of Qom university of medical sciences under the ethics code (IR.MUQ.REC.1394.47).

\section{Specimen collection}

All patients were enrolled after consenting for participation. Stool samples (1-2 gr) were collected inside a clean, dry and waterproof container with no detergent. Peripheral blood samples were taken for laboratory evaluation including CBC, ESR and CRP at the time of enrollment. All data were recorded in unified data collection forms.

\section{Rotavirus Detection}

Fifty mgs of a solid sample or two drops of liquid sample (equivalent to $50 \mathrm{mg}$ ) were transferred into the test tube. The tube was capped and shaken for two minutes to ensure dissolution into buffer liquid. Then two drops (equivalent to $80 \mathrm{cc}$ ) of the sample was loaded on the diagnostic kit making sure no bubbles were formed. Finally, the Rotavirus antigen was added to the kit and the test result was read after five minutes. The results were reported within 10 minutes and were considered invalid if not read within 20 minutes of loading. The test was considered as positive for Rotavirus if both $\mathrm{C}$ and $\mathrm{R}$ lines were illuminated. The test result was considered invalid if the $C$ line is not there. 


\section{Statistical Analysis}

The data were analyzed using SPSS software (version 22). The mean and standards deviation were calculated for continuous variables. Absolute and relative abundance were counted for the numerical variables. The chi-squared, Fisher's exact test and t-test were used to assess the relationship between clinical and laboratory features with rotavirus positivity. A p-value less than 0.05 was considered statistically significant.

\section{Results}

One hundred and ninety-nine children were recruited to the study including 118 boys (59.3\%) and 81 $(40.7 \%)$ girls. The mean age of children was 3.3 year (1-15 year). Sixty-nine (31.7\%) of patients received antibiotic treatment. The prevalence of Rotavirus-associated diarrhea was $20.1 \%$ among children hospitalized with diarrhea.

The prevalence of the infection was $22.8 \%$ and $16 \%$ among boys and girls respectively; this difference was not statistically significant $(p=0.23)$.

One-hundred-and-nineteen (59.8\%) of 199 participants were hospitalized for diarrhea in spring being in prevalence followed by $47(23.6 \%)$ cases in summer, and 33(16.6\%) cases in winter. The seasonal distribution of the Rotavirus-associated diarrhea was $15.9 \%$ in spring, $21.2 \%$ in summer, and $33.3 \%$ in winter; with no significant difference across seasons $(p=0.086)$.

Table 1

Comparison of Mean Age and Laboratory Markers between study groups

\begin{tabular}{|lll|}
\hline Variable & $\begin{array}{l}\text { Rotavirus-associated diarrhea } \\
\text { Mean( } \pm \text { SD) }\end{array}$ & $\begin{array}{l}\text { Rota-virus negative diarrhea } \\
\text { Mean }( \pm \text { SD })\end{array}$ \\
\hline Age(years $)$ & $2.3(1.5)$ & $3.5(2.6)$ \\
\hline WBC $\left(\times 10^{9} / \mathrm{L}\right)$ & $9.4(2.5)$ & $11.19(4.7)$ \\
\hline PMN $(\%)$ & $49.7 \%(12.8)$ & $58.4 \%(14.9$ \\
\hline CRP $(\mathrm{mg} / \mathrm{L})$ & $16(15)$ & $29.2(24)$ \\
\hline ESR & $19.8(13.6)$ & $24.9(17.4)$ \\
\hline
\end{tabular}

The mean WBC count was significantly higher among diarrhea patients negative for rotavirus infection compared to patients with rotavirus-associated diarrhea $(p=0.02)$. Also, children with rotavirus-positive diarrhea showed lower mean PMN, CRP, and ESR compared to patients who tested negative $(T a b l e 1)(p<$ 0.05). Patients with a positive Rotavirus antigen test were also more likely to have RBC or WBC in their stool samples(p-value $<0.05)$. 


\section{Discussion}

We found that 0.20 (95\% $\mathrm{Cl} 0.14-0.26)$ of collected stool samples from hospitalized children with diarrhea were positive for Rotavirus. This is considerably lower than previous studies from Iran. A 2014 systematic review on prevalence of Rotavirus infection in Iran showed that Rotavirus infection rates for hospitalized children were slightly higher than outpatients(5). They reported an overall proportion of 0.35 (95\% Cl, 0.28-0.41) among Iranian children with gastroenteritis with a wide variation between 11.36$79 \%(5)$. A 2017 meta-analysis showed a higher overall estimate of about 39\%(14). One reason for the difference in our results compared to the overall rates in Iran may be due to the regional epidemiologic differences. Interestingly, low rates of Rotavirus causality in children's diarrhea have been previously reported in central regions of Iran such as Tehran and Esfahan; This in line with the low rate in our study may raise the possibility that the central region of Iran may have lower rates of Rotavirus infection.

Notably, studies from Iran used different techniques in viral isolation and detection from stool samples. This has introduced significant variations in the rates of rotavirus infection across studies owing to difference in accuracy of these methods(1). We detected (20.1\%) of diarrhea patients with Rotavirus infection using EIA test although the virus was not found in association with age and gender of the patients.

Also, our study population had a wider age range (1-15 years) than most of previous studies that only included patients younger than 5 years. This factor as well as the decreased chance of RV-related diarrhea as children get older, may account for the apparently lower rate of Rotavirus infection in the current study population.

In spite of the wide age variation in our population, the mean age of patients in the rotavirus-positive group was 2.3 years which was lower than the mean age of the sample. Based on this finding which is consistent with previous findings, we postulate that the likelihood of rotavirus infection is most common in children $2-3$ years of age $(5,15,16)$.

While most studies have shown a higher rate of Rotavirus infections in colder and dryer seasons with the peak rate happening in Winter(7, 17-19), the Gulf region countries including Iran are an exception showing no distinct peak(20). Our results showed a higher albeit non-significant rate of rotavirus positivity in Winter (33\%) among children hospitalized with acute diarrhea without any clear peaks. This lack of significant variation may be due to the hot desert climate in Qom with low rate of rainfalls. The same factor may account for the lower rate found in this study compared with other regions of Iran.

We found significantly lower levels of CRP, ESR, and WBC in patients with rotavirus-related diarrhea compared to rotavirus-negative children. This finding may be due to the higher rate of bacterial infections in rotavirus-negative children hospitalized for diarrhea.

Our study was limited by several factors: we used antigen detection method which has sub-optimal accuracy and did not assess for the genotypic variation of Rotavirus in our population. Also, we did not 
assess for the etiology of diarrhea in non-rotavirus cases making judgement about the difference in laboratory markers difficult.

\section{Conclusion}

Our research showed a relatively low rate of rotavirus infection among children hospitalized with acute GE in Qom. The majority of cases involved children 2-3 years old. Also, patients with positive rotavirus test were more likely to have microscopically abnormal stool exams. Although the rates of rotavirus infection were not high in our study, we recommend further research with more precise laboratory methods and genotyping to guide decisions on vaccination strategies.

\section{Abbreviations}

RNA

Ribonucleic acid; WHO:World Health Organization; ELISA:enzyme-linked immunosorbent assay; LA:latex agglutination; EM:electron microscopy; $\mathrm{CBC}$ :complete blood count; ESR:erythrocyte sedimentation rate; CRP:C-reactive protein; Ag:Antigen; SD:standard deviation; PMN:polymorphonuclear; WBC:white blood cell; RBC:Red blood cell; Cl:confidence interval; EIA:enzyme-linked immunosorbent assay.

\section{Declarations}

Ethics approval and consent to participate: All patients were enrolled into the study after taking an informed consent. The study protocol was approved at ethics committee of Qom university of medical sciences under the ethics code (IR.MUQ.REC.1394.47).

Consent for publication: All authors have read and agreed to publish the present manuscript.

Availability of data: The data obtained in the present study are fully presented in the manuscript.

Competing interests: The authors declare that there is no conflict of interest.

Funding: The author(s) received no financial support for the research, authorship, and/or publication of this article.

Authors' contributions: M.A. Khajeh-Azad, S. Rezvan and S. Shams contributed to data collection, data analysis and to the writing of the manuscript. $\mathrm{H}$. Heydari contributed to the design and implementation of the research. M. Aghaali and Z. Movahedi contributed to design and data collection.

\section{Acknowledgements: None.}

\section{References}

1. Badur S, Öztürk S, Pereira P, AbdelGhany M, Khalaf M, Lagoubi Y, et al. Systematic review of the rotavirus infection burden in the WHO-EMRO region. Human vaccines \& immunotherapeutics. 2019. 
2. Kawai K, O’Brien MA, Goveia MG, Mast TC, El Khoury AC. Burden of rotavirus gastroenteritis and distribution of rotavirus strains in Asia: a systematic review. Vaccine. 2012;30(7):1244-54.

3. Parashar UD, Hummelman EG, Bresee JS, Miller MA, Glass RI. Global illness and deaths caused by rotavirus disease in children. Emerging infectious diseases. 2003;9(5):565.

4. Incecik F, Hergüner MO, Altunbaşak Ş, Solgun H. Acute encephalopathy associated rotavirus gastroenteritis. Journal of pediatric neurosciences. 2009;4(2):141.

5. Moradi-Lakeh M, Shakerian S, Yaghoubi M, Esteghamati A, Shokraneh F, Baradaran HR, et al. Rotavirus Infection in Children with Acute Gastroenteritis in Iran: A Systematic Review and Metaanalysis. International journal of preventive medicine. 2014;5(10):1213-23.

6. Bányai K, László B, Duque J, Steele AD, Nelson EAS, Gentsch JR, et al. Systematic review of regional and temporal trends in global rotavirus strain diversity in the pre rotavirus vaccine era: insights for understanding the impact of rotavirus vaccination programs. Vaccine. 2012;30:A122-A30.

7. Lestari FB, Vongpunsawad S, Wanlapakorn N, Poovorawan Y. Rotavirus infection in children in Southeast Asia 2008-2018: disease burden, genotype distribution, seasonality, and vaccination. Journal of biomedical science. 2020;27:1-19.

8. Troeger C, Khalil IA, Rao PC, Cao S, Blacker BF, Ahmed T, et al. Rotavirus Vaccination and the Global Burden of Rotavirus Diarrhea Among Children Younger Than 5 Years. JAMA Pediatr. 2018;172(10):958-65.

9. Jonesteller CL, Burnett E, Yen C, Tate JE, Parashar UD. Effectiveness of rotavirus vaccination: a systematic review of the first decade of global postlicensure data, 2006-2016. Clinical Infectious Diseases. 2017;65(5):840-50.

10. Lamberti LM, Ashraf S, Walker CLF, Black RE. A systematic review of the effect of rotavirus vaccination on diarrhea outcomes among children younger than 5 years. The Pediatric infectious disease journal. 2016;35(9):992-8.

11. Kotloff KL, Nataro JP, Blackwelder WC, Nasrin D, Farag TH, Panchalingam S, et al. Burden and aetiology of diarrhoeal disease in infants and young children in developing countries (the Global Enteric Multicenter Study, GEMS): a prospective, case-control study. The Lancet. 2013;382(9888):209-22.

12. Khosravi AD, Mihani F. Detection of metallo-beta-lactamase-producing Pseudomonas aeruginosa strains isolated from burn patients in Ahwaz, Iran. Diagn Microbiol Infect Dis. 2008;60(1):125-8.

13. Kargar M, Akbarizadeh AR. Prevalence and molecular genotyping of group A rotaviruses in Iranian children. Indian Journal of Virology. 2012;23(1):24-8.

14. Monavari SHR, Hadifar S, Mostafaei S, Miri A, Keshavarz M, Babaei F, et al. Epidemiology of rotavirus in the Iranian children: A systematic review and meta-analysis. Journal of global infectious diseases. 2017;9(2):66.

15. Shrestha S, Thakali O, Raya S, Shrestha L, Parajuli K, Sherchand JB. Acute gastroenteritis associated with Rotavirus A among children less than 5 years of age in Nepal. BMC infectious diseases. 2019;19(1):1-8. 
16. Kargar M, Javdani N, Najafi A, Tahamtan Y. First molecular detection of group A rotavirus in urban and hospital sewage systems by nested-RT PCR in Shiraz, Iran. Journal of Environmental Health Science and Engineering. 2013;11(1):1-6.

17. Patel MM, Pitzer V, Alonso WJ, Vera D, Lopman B, Tate J, et al. Global seasonality of rotavirus disease. The Pediatric infectious disease journal. 2013;32(4):e134.

18. Jagai JS, Sarkar R, Castronovo D, Kattula D, McEntee J, Ward H, et al. Seasonality of rotavirus in South Asia: a meta-analysis approach assessing associations with temperature, precipitation, and vegetation index. PloS one. 2012;7(5):e38168.

19. Levy K, Hubbard AE, Eisenberg JN. Seasonality of rotavirus disease in the tropics: a systematic review and meta-analysis. International journal of epidemiology. 2009;38(6):1487-96.

20. Tayeb HT, Dela Cruz DM, Al-Qahtani A, Al-Ahdal MN, Carter MJ. Enteric viruses in pediatric diarrhea in Saudi Arabia. Journal of medical virology. 2008;80(11):1919-29. 